\title{
Research of the basic characteristics of the new magnetic elastic force sensors
}

\author{
Sulton Amirov ${ }^{1}$, Kamila Jurayeva ${ }^{1}$ \\ ${ }^{1}$ Tashkent institute of railway engineering, Department of Power Supply of Railways, Adilkhodjayev - 1, \\ Tashkent, 100167, Uzbekistan
}

\begin{abstract}
The article investigated the static, dynamic and metrological characteristics of new magnetoelastic force sensors taking into account the distribution of parameters of electrical and magnetic circuits. It is shown that taking into account the distribution of circuit parameters during calculations significantly reduces the error in calculating the characteristics of magnetoelastic sensors.
\end{abstract}

\section{Introduction}

All over the world, research work is underway aimed at developing new power measuring elements and devices for monitoring and control systems with high sensitivity, accuracy and linearity of conversion characteristics, high reliability and stability of characteristics under extreme operating conditions. At the same time, magnetoelastic force sensors (MFS) are the most fully meeting the requirements of monitoring and control systems.

\subsection{New magnetoelastic force sensors}

New designs of MFS have been developed [1,2]. It is shown that in a MFS containing coaxially located annular magnetic circuits with sections of measuring windings and through slots made along the circumference, dividing each annular magnetic circuit into two rods, elastic conical bushings located coaxially with the annular magnetic circuits and interacting with the bases with the surfaces of the annular magnetic circuits, performing ring magnetic cores are identical and the introduction of additional, concentrically and mutually mirrored elastic conical bushings, lips Installed with the possibility of simultaneous interaction with the inner and outer surfaces of the annular magnetic cores, as well as the inclusion of the measuring windings of the annular magnetic cores with each other in series, increases the sensitivity, accuracy and linearity of the sensor conversion characteristics. In addition, it has been shown that the implementation of through slots on the forming surfaces of the annular magnetic cores and the reinforcement of the horseshoe-shaped ring casings of inelastic material on the inner and outer forming surfaces of the annular magnetic cores, on which the bases of the conical elastic bushings act, increases the sensitivity of the MFS.

\subsection{MFS magnetic circuits}

The expressions of magnetic fluxes in coaxially arranged annular magnetic cores and magnetic voltages between them are determined depending on the length of the magnetic circuit, taking into account the distributed nature of the magnetic resistance of the magnetic circuit, the magnetic capacity of the annular gap and sections of the magnetizing windings (Fig. 1).

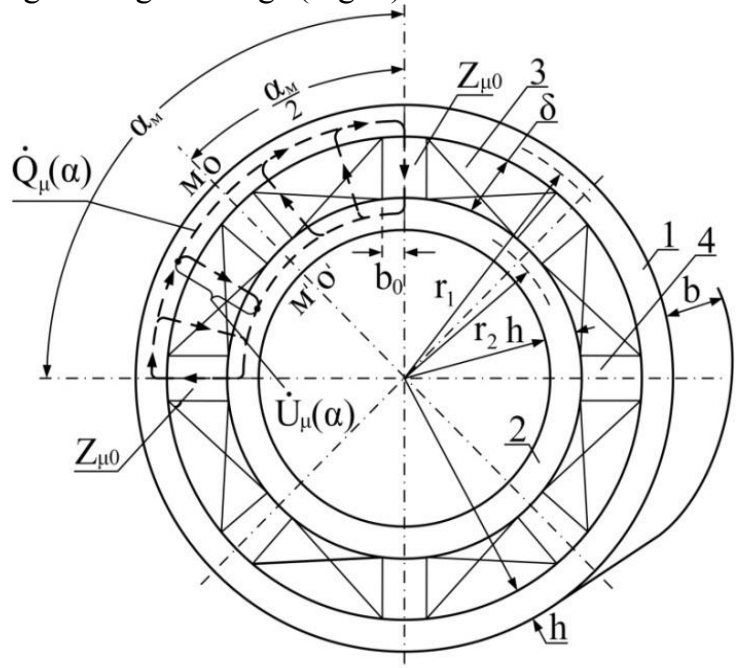

Fig. 1. The design of the magnetic circuit developed by the MFS

1, 2 - coaxially located annular magnetic cores; 3 - section magnetizing windings; 4 - ferromagnetic jumpers; $r_{1}, r_{2}, h, b$ - respectively, the average radius, thickness and height of the annular magnetic circuits 1 and $2 ; \delta$ - air gap between the ring magnetic circuits; $b_{0}$ - width of the ferromagnetic jumper.

In order to simplify the analysis of the magnetic circuit of the MFS under study, we neglect the lateral scattering fluxes and assume that the ferromagnetic jumpers with the corresponding sections of the magnetizing winding are identical. In addition, we 
assume that the magnetic circuit of the investigated MFS operates on a linear section of the main magnetization curve of the magnetic circuit material. These assumptions, as shown by experimental studies, introduce minor inaccuracies in the calculations, but significantly simplify the analysis of the magnetic circuit under study $[3,4]$.

In the study of the magnetic circuits of the new MFS, the method of constructing an equivalent circuit (Fig. 2) and the classical method of compiling and solving differential equations were used [5].

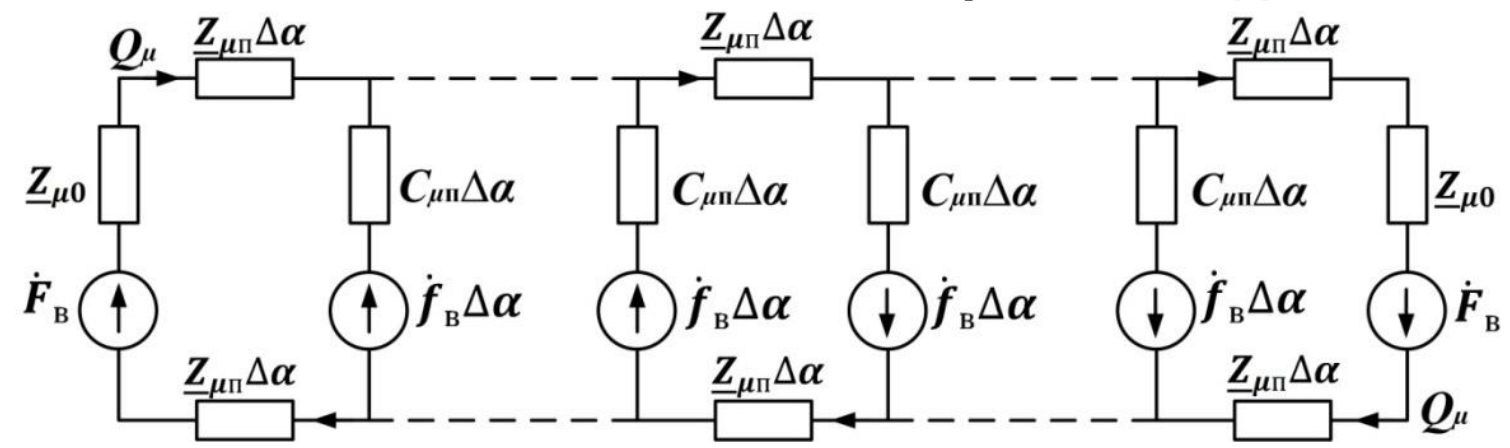

Fig. 2. Magnetic circuit equivalent circuit taking into account the distribution of sections of the magnetizing winding

Expressions for magnetic voltage and magnetic flux of magnetic circuits developed by MFS with concentrated sections of magnetizing windings:

$$
\begin{aligned}
\dot{U}_{\mu}(\alpha) & =\frac{\dot{F}_{\mathrm{B}}}{\underline{\Delta}}\left\{\left\{\operatorname{sh}\left[\underline{\gamma}\left(\alpha_{\mathrm{M}}-\alpha\right)\right]-\operatorname{sh}(\underline{\gamma} \alpha)\right\}+\right. \\
& \left.+\frac{\underline{\underline{z}} \underline{\underline{Z}}_{\mu 0}}{2 \underline{Z}_{\mu \mathrm{\Pi}}}\left\{\operatorname{ch}\left[\underline{\gamma}\left(\alpha_{\mathrm{M}}-\alpha\right)\right]-\operatorname{ch}(\underline{\gamma} \alpha)\right\}\right\}, \\
\dot{Q}_{\mu}(\alpha)= & \frac{\underline{\gamma} \dot{F}_{\mathrm{B}}}{2 \underline{Z}_{\mu \mathrm{\Pi}} \underline{\Delta}}\left\{\left\{\operatorname{ch}\left[\underline{\gamma}\left(\alpha_{\mathrm{M}}-\alpha\right)\right]++\operatorname{ch}(\underline{\gamma} \alpha)\right\}+\right. \\
& \left.+\frac{\underline{\gamma} \underline{\underline{Z}}_{\mu \mathrm{0}}}{2 \underline{Z}_{\mu \mathrm{n}}}\left\{\operatorname{sh}\left[\underline{\gamma}\left(\alpha_{\mathrm{M}}-\alpha\right)\right]+\operatorname{sh}(\underline{\gamma} \alpha)\right\}\right\} .
\end{aligned}
$$

where is $\dot{F}_{\mathrm{B}}$ - the magnetomotive force (MMF) of the magnetizing winding section; $\underline{\gamma}=\sqrt{2 \underline{Z}_{\mu \Pi} C_{\mu \Pi}}$ - the complex value of the coefficient of propagation of the magnetic flux along the magnetic circuit, $\frac{1}{\text { grad }} ; \underline{Z}_{\mu \text { п }}=$ $\frac{1}{\dot{\mu}_{\text {б. }} \mu_{0} h b}, \quad C_{\mu \text { п }}=\mu_{0} \frac{b}{\delta}$ - linear values of the complex magnetic resistance of the ring magnetic circuits and the magnetic capacity of the annular gap per unit angle of the magnetic circuit; $\quad \underline{\Delta}=\left(1+\frac{\underline{\gamma}^{2} \underline{Z}_{\mu 0}^{2}}{4 \underline{Z}_{\mu \mathrm{I}}^{2}}\right) \operatorname{sh}\left(\underline{\gamma} \alpha_{\mathrm{M}}\right)+$ $+\frac{\underline{\gamma} \underline{Z}_{\mu 0}}{\underline{Z}_{\mu \text { п }}} \operatorname{ch}\left(\underline{\gamma} \alpha_{\mathrm{M}}\right) ; \underline{Z}_{\mu 0}$ - complex magnetic resistance of ferromagnetic jumpers, $1 / \mathrm{Gn} ; \dot{\mu}_{\text {б.п. }}$ - the relative magnetic permeability of the ferromagnetic material without taking into account energy losses; $\alpha_{\mathrm{M}}$ maximum value of the angular coordinate $\alpha$.

Expressions for magnetic voltage and magnetic flux of magnetic circuits developed by MFS with distributed sections of magnetizing windings:

$$
\begin{aligned}
& \dot{U}_{\mu}(\alpha)=\frac{\dot{F_{\mathrm{B}}}}{\underline{\Delta}}\left\{\operatorname{sh}\left[\underline{\gamma}\left(\alpha_{\mathrm{M}}-\alpha\right)\right]-\operatorname{sh}(\underline{\gamma} \alpha)\right\}+\dot{F}_{\mathrm{B}} \frac{\underline{\gamma} \underline{Z}_{\mu 0}}{2 \underline{Z}_{\mu \mathrm{n}} \underline{\Delta}} . \\
& \cdot\left\{\operatorname{ch}\left[\underline{\gamma}\left(\alpha_{\mathrm{M}}-\alpha\right)\right]-\operatorname{ch}(\underline{\gamma} \alpha)\right\}+\dot{f}_{\mathrm{B}} \frac{\underline{Z}_{\mu 0}}{2 \underline{Z}_{\mu \mathrm{\Pi}} \underline{\Delta}} \cdot \\
& \cdot\left\{\operatorname{sh}\left[\underline{\gamma}\left(\alpha_{\mathrm{M}}-\alpha\right)\right]-\operatorname{sh}(\underline{\gamma} \alpha)\right\}+\dot{f}_{\mathrm{B}} \frac{\underline{\gamma} \underline{Z}_{\mu 0}^{2}}{4 \underline{Z}_{\mu \mathrm{\Pi}}^{2} \underline{\Delta}} \text {. } \\
& \cdot\left\{\operatorname{ch}\left[\underline{\gamma}\left(\alpha_{\mathrm{M}}-\alpha\right)\right]-\operatorname{ch}(\underline{\gamma} \alpha)\right\} \\
& \dot{Q}_{\mu}(\alpha)=\frac{\underline{\gamma} \dot{F}_{\mathrm{B}}}{2 \underline{Z}_{\mu \Pi} \underline{\Delta}}\left\{\operatorname{ch}\left[\underline{\gamma}\left(\alpha_{\mathrm{M}}-\alpha\right)\right]+\operatorname{ch}(\underline{\gamma} \alpha)\right\}+ \\
& +\dot{F}_{\mathrm{B}} \frac{\underline{\gamma}^{2} \underline{Z}_{\mu 0}}{4 \underline{Z}_{\mu \mathrm{n}}^{2} \underline{\Delta}}\left\{\operatorname{sh}\left[\underline{\gamma}\left(\alpha_{\mathrm{M}}-\alpha\right)\right]+\operatorname{sh}(\underline{\gamma} \alpha)\right\}+ \\
& +\dot{f}_{\mathrm{B}} \frac{\underline{\gamma} \underline{Z}_{\mu 0}}{4 \underline{Z}_{\mu \mathrm{\Pi}}^{2} \underline{\Delta}}\left\{\operatorname{ch}\left[\underline{\gamma}\left(\alpha_{\mathrm{M}}-\alpha\right)\right]+\operatorname{ch}(\underline{\gamma} \alpha)\right\}+ \\
& +\dot{f}_{\mathrm{B}} \frac{\underline{\gamma}^{2} \underline{Z}_{\mu 0}^{2}}{8 \underline{\Delta \Delta Z_{\mu \Pi}}}\left\{s h\left[\underline{\gamma}\left(\alpha_{\mathrm{M}}-\alpha\right)\right]+\operatorname{sh}(\underline{\gamma} \alpha)\right\}-\frac{\dot{f}_{\mathrm{B}}}{2 \underline{Z}_{\mu \pi}}
\end{aligned}
$$

here $\dot{f}_{\mathrm{B}}=$ const - is the linear value of the magnetizing force of a uniformly distributed section of the field winding per unit angle $\alpha$.

An analysis of equations (1) - (4) and their curves (Fig. 3) shows that magnetic the stresses along the MFS magnetic circuit of the efforts, respectively, with concentrated and distributed magnetizing forces are distributed nonlinearly and change their signs at the magnetic neutral point, and the corresponding magnetic fluxes are unstable and have a minimum value at the magnetic neutral point, and with increasing magnetic flux $\beta$ attenuation coefficient the degree of nonlinearity of the magnetic voltage distribution and the inconstancy of the magnetic flux along the length of the magnetic circuit increases. 


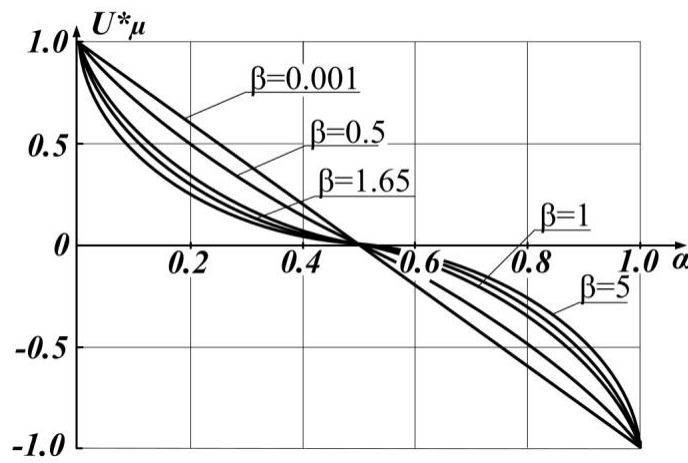

a)

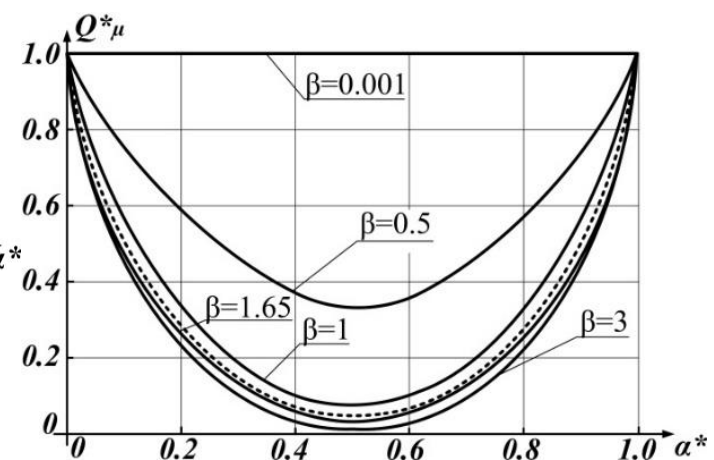

b)

Fig. 3. Dependence curves $U_{\mu}^{*}=f\left(\alpha^{*}\right)$ (a) and $Q_{\mu}^{*}=f\left(\alpha^{*}\right)$ (b) for different values of the magnetic flux attenuation coefficient $\beta$ : solid curves are -calculated and dashed curves are - experimental data; $U_{\mu}^{*}\left(\alpha^{*}\right)=\frac{U_{\mu}\left(\alpha^{*}\right)}{U_{\mu}(0)} ; Q_{\mu}^{*}\left(\alpha^{*}\right)=\frac{Q_{\mu}\left(\alpha^{*}\right)}{Q_{\mu}(0)} ; \alpha^{*}=\frac{\alpha}{\alpha_{\mathrm{M}}}$.

A comparative analysis of the calculated and experimental dependence curves $U_{\mu}^{*}=f\left(\alpha^{*}\right)$ and $Q_{\mu}^{*}=$ $f\left(\alpha^{*}\right)$ magnetic circuits, respectively, without taking into account and taking into account the distribution of magnetizing forces, showed that taking into account the distribution of the magnetizing forces of the excitation coil sections significantly (up to $20 \%$ ) reduces the error in calculating the magnetic circuits of the MFS forces.

Note that expressions (1) - (4) are mathematical models of magnetic circuits developed by MFS.

\subsection{Static characteristics of new MFS}

The analytical equation of the static characteristic of the magnetoelastic transformation stage, i.e. the dependence of the relative magnetic permeability of the magnetic circuit developed by MFS on external mechanical stresses and magnetic field strength created by the excitation winding with alternating current, obtained in the form of the following approximating function [6]:

$$
\mu_{\text {б.п. }}=a_{1} \pm a_{2} \sigma
$$

where, $a_{1}, a_{2}$ are the approximation coefficients; $\sigma$ - the absolute value of compressive mechanical stresses. Dependency approximation $\mu_{\text {б.п. }}=f(\sigma)$ for electrical steel E3 in the initial section of the main magnetization curve gave the following values of the approximation coefficients: $a_{1}=1085$ and $a_{2}=9,77 \mathrm{Mm}^{2} \cdot \mathrm{N}^{-1}$.

In the fig. 4 shown the dependency graphs $\mu=$ $f\left(U_{\mathrm{M}}\right)$ and $\Delta \mu=f\left(U_{\mathrm{M}}\right), U_{\mathrm{M}}$ - is the mechanical force. An analysis of the obtained analytical and experimental dependences of the relative magnetic permeability of the MFS ferromagnetic core on the applied mechanical stresses shows that for MFS whose magnetic circuit operates on a linear portion of the main magnetization curve, the above dependence can be approximated as a linear function with a negative (positive) coefficient, sign which depends on the brand used of magnetoelastic material.

The expression of the output signal at the output of one concentrated section of the measuring winding with the concentrated section of the magnetizing winding for the developed MFS has the following form:

$$
\begin{aligned}
& E_{\text {out }}=2 j \omega W_{\text {out }} \frac{\underline{\beta}\left(U_{\mathrm{M}}\right) \dot{F}_{\mathrm{B}}}{\underline{Z}_{\mu_{\mathrm{\Pi}}}\left(U_{\mathrm{M}}\right) \alpha_{\mathrm{M}} \underline{\Delta}^{*}}\left[\operatorname{ch}\left(\frac{1}{2} \underline{\beta}\left(U_{\mathrm{M}}\right)\right)+\right. \\
& \left.+\underline{\beta}\left(U_{\mathrm{M}}\right) \underline{k}_{0} \operatorname{sh}\left(\frac{1}{2} \underline{\beta}\right)\right] \operatorname{ch}\left[\left(\frac{1}{2} \underline{\beta}\left(U_{\mathrm{M}}\right)\right)\right],
\end{aligned}
$$

(6)

where $W_{\text {out }}$ - is the number of turns of a uniformly distributed measuring winding.

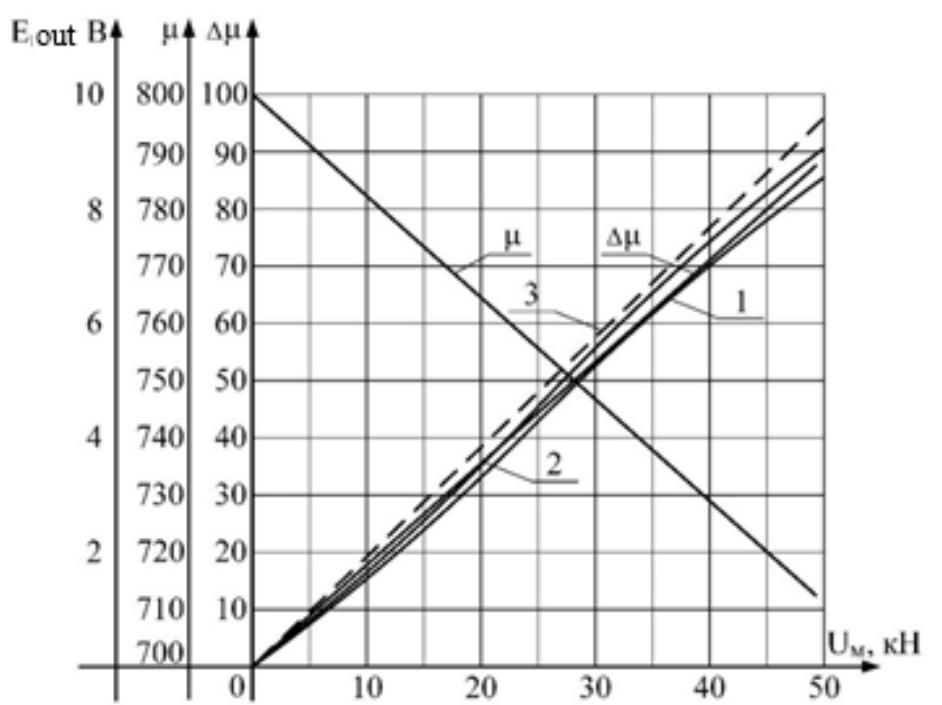

Fig. 4. Dependency graphs $\mu=f\left(U_{\mathrm{M}}\right), \Delta \mu=f\left(U_{\mathrm{M}}\right)$ and $E_{\text {out }}=f\left(U_{\mathrm{M}}\right): 1$ - without taking into account the distributed nature of the windings; 2 - taking into account the distributed nature of the windings; 3 - experimental characteristic 
The expression of the output signal at the output of one distributed section of the measuring winding with the distributed section of the magnetizing winding has the following form:

$$
\begin{aligned}
& E_{\text {out }}=2 j \omega W_{\text {out sp. }}\left\{\frac{\dot{F}_{\mathrm{B}}}{2 \underline{Z}_{\mu \mathrm{\Pi}}\left(U_{\mathrm{M}}\right) \alpha_{\mathrm{M}}}-\frac{\underline{\beta}\left(U_{\mathrm{M}}\right)}{2 \underline{Z}_{\mu \Pi} \alpha_{\mathrm{M}}}\left(2 \frac{\dot{F}_{\mathrm{B}}}{\underline{\Delta}^{*}}+\right.\right. \\
& \left.+\dot{f}_{\mathrm{B}} \frac{\alpha_{\mathrm{M}} \underline{k}_{0}}{\underline{\Delta^{*}}}\right) \cdot\left[\operatorname{ch}\left(\frac{1}{2} \underline{\beta}\left(U_{\mathrm{M}}\right)\right)+\underline{\beta}\left(U_{\mathrm{M}}\right) \underline{k}_{0} \operatorname{sh}\left(\frac{1}{2} \underline{\beta}\left(U_{\mathrm{M}}\right)\right)\right] . \\
& \cdot \operatorname{sh}\left(\frac{1}{2} \underline{\beta}\left(U_{\mathrm{M}}\right)\right\}
\end{aligned}
$$

where $W_{\text {out sp. }}$ - is the specific value of the number of turns of a uniformly distributed measuring winding.

\section{MFS}

\subsection{Dynamic characteristics of the developed}

The dynamic characteristics of the developed MFS were studied under the following six typical modes (influences): 1) a constant force acts on the MFS and it is connected to a sinusoidal voltage source; 2) MFS is connected to a constant voltage source, and a single force is applied to the sensor input; 3) MFS is connected to a constant voltage source, and a linearly increasing force is applied to the sensor input; 4) MFS is connected to a sinusoidal voltage source, and a single force is applied to the sensor input; 5) with a sinusoidal voltage of the power source and supplying a linearly increasing force to the sensor input; 6) with a sinusoidal voltage of the power source and supply to the input of the sensor a sinusoidal force [7]. Expressions of the transient voltage are obtained for these modes, and for the first mode and expressions of the amplitude and phase-frequency characteristics. In particular, for the fourth mode, the expression has the form (Fig. 5):

$$
\begin{aligned}
U_{\text {э.out }}(t) & =U_{\text {эп }}(t) \frac{k_{2} U_{\mathrm{M}}}{T_{\ni \mu}} e^{-\frac{t}{T_{\ni} \mu}}= \\
= & \frac{k_{2} U_{\mathrm{M}} U_{\ni п m}}{T_{\ni} \mu} e^{-\frac{t}{T_{\ni} \mu}} \sin \omega_{\ni} t .
\end{aligned}
$$

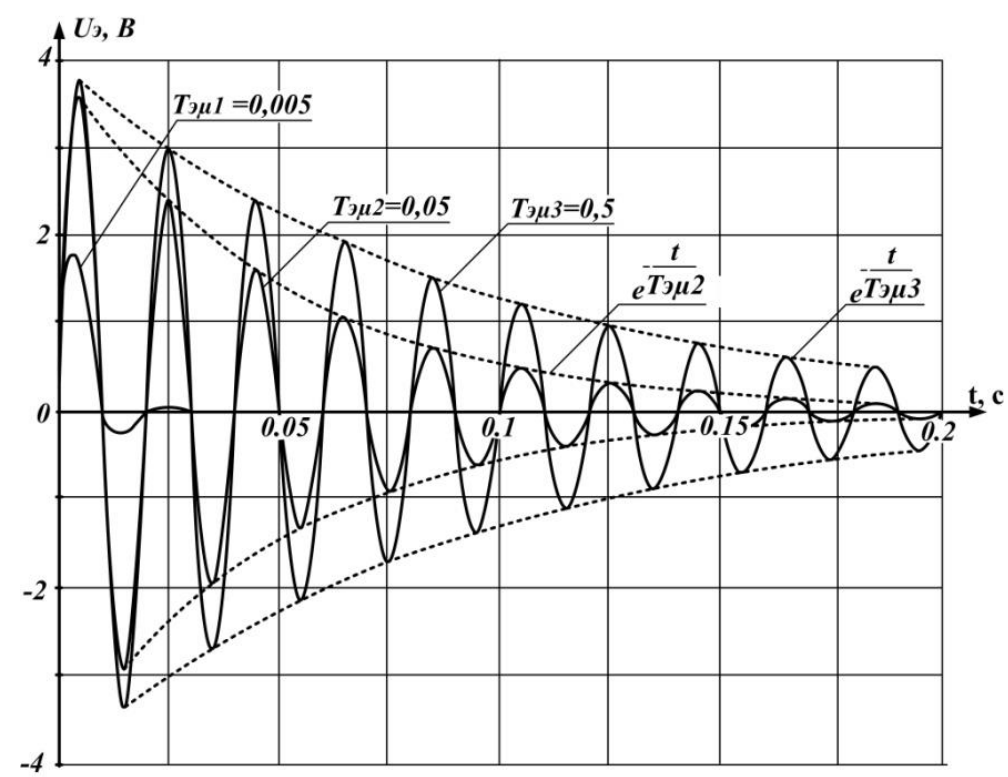

Fig.5. Transient voltage curves for the mode when the developed MFS is connected to a sinusoidal voltage source, and a single force is applied to the sensor input

where $\quad k_{2}=G_{\ni} K_{I_{\ni} U_{\mu}} K_{I_{\mu} U_{\ni}} K_{U_{\mathrm{M}} C_{\mu}} ; \quad G_{\ni} \quad-\quad$ electrical conductivity of the magnetization circuit; $K_{I_{3} U_{\mu}}=w_{\text {inp }}$ the number of turns of the input winding is the conversion coefficient of the interchain physical and technical effect (PTE) between the electric current $I_{\ni}$ and the magnetic voltage $U_{\mu} ; K_{I_{\mu} U_{\ni}}=w_{\text {out }}$ - the number of turns of the input (measuring) winding - the conversion coefficient of the inter-circuit PTE of electromagnetic mutual induction between the magnetic current $I_{\mu}$ and the output electrical voltage $U_{\text {out }} ; K_{U_{\mathrm{M}} C_{\mu}}$ - the coefficient of interchain PTE (magnetoelastic effect) between the mechanical force $U_{\mathrm{M}}$ and magnetic capacity (magnetic conductivity by the classical analogy of chains); $T_{\ni \mu}=$ $T_{\ni}+T_{\mu}$ - time constant of the electromagnetic circuit developed by MFS; $T_{\mu}=R_{\mu} C_{\mu \Sigma}$ and $T_{\ni}=$
$G_{\ni} K_{I_{\ni} U_{\mu}} K_{I_{\mu} U_{\ni}} C_{\mu \Sigma}=w_{i n p}^{2} C_{\mu \Sigma} G_{\ni}=\frac{L_{\ni} i n p}{R_{\ni}}$ - respectively, the time constant of the magnetic and electrical circuits, $L_{\text {э.inp }}$ - electrical inductance of the excitation circuit; $C_{\mu \Sigma}$ - the total magnetic capacity of the magnetic circuit in the path of the working magnetic flux $Q_{\mu} ; U_{\text {эпт }}$ and $\omega_{\ni}$ respectively, the amplitude and frequency of the voltage of the power source.

An analysis of the obtained expressions and their curves for dynamic modes showed that the developed MFS in the monitoring and control system correspond to real differentiating links with statism, and at high frequencies of the voltage of the power source, i.e. when $\omega_{\ni} T_{\ni \mu} \gg 1$, MFS from a differentiating link turns into a proportional one. 


\subsection{The error of the new MUD}

The analysis of the compiled classification of possible sources of errors developed by the MFS showed that methodological, technological, operational errors are systematic sources, and internal, external and operational errors are sources of random errors. To identify the sources of errors and their analysis, the apparatus of parametric structural schemes was used, as well as the well-known concepts of additive and multiplicative errors $[8,9]$. A quantitative assessment of errors showed that the non-linearity of the operating characteristic (the error is up to $\pm 7.0 \%$ ), magnetoelastic hysteresis (up to $\pm 2.0 \%$ ), changes in the ambient temperature (up to $\pm 2.5 \%)$ and the instability of the parameters of the power supply (up to $\pm 15.0 \%$ ). It has been found that the most profitable ways to reduce the error: namely, from the nonlinearity of the operating characteristic of the MUD is the conversion of the analog signal to a proportional frequency (the error decreases to $\pm 0.1 \%$ ); from glueelastic hysteresis - production of a magnetic circuit monolithic (up to $\pm 0.2 \%$ ); from changes in ambient temperature - the use of a second, stress-free MFS (up to $\pm 0,1 \%$ per $10^{\circ} \mathrm{C}$ of its additive component) and the use of differential circuits of two MFS, loaded with forces of different signs (compression and tension) (up to \pm 0.015 per $10^{\circ} \mathrm{C}$ of its multiplicative component); from instability of power supply parameters - conversion of highly stable DC voltage into rectangular pulses (up to $\pm 0.01 \%$ ). Calculations and experiments have shown that the relative reduced error of the developed MFS does not exceed $\pm 0.5 \%$. The main characteristics of the developed MFS shown in Table 1.

Table 1. Main characteristics of the developed MFS

\begin{tabular}{|l|l|}
\hline Range of the measured efforts, $\mathrm{kN}$ & $5 \cdot 10^{2} \div 5 \cdot 10^{4}$ \\
\hline Sensitivity, $\mathrm{mV} / \mathrm{kN}$ & 0.2 \\
\hline Conversion error, $\%$ & 0.5 \\
\hline Nonlinearity, $\%$, no more than & 0.5 \\
\hline Range of output voltage variation, $\mathrm{V}$ & $0 \div 10$ \\
\hline Operating temperature range, ${ }^{\circ} \mathrm{C}$ & $-30 \ldots+60$ \\
\hline Speed, s & $10^{-4}$ \\
\hline Overall dimensions, mm & $120 \mathrm{x} 100 \mathrm{x} 100$ \\
\hline Weight, kg, no more than & 0.5 \\
\hline
\end{tabular}

\section{Conclusion}

Thus, the main technical characteristics of the new magnetoelastic force sensors taking into account the distribution of the parameters of their electric and magnetic circuits are investigated in the article.

\section{References}

1. Patent RUz (UZ) №IAP 04866. Magnitouprugiy datchik usiliy/ Amirov S.F., Nazirova Z.G., Jurayeva K.K., Boltaev O.T., Sharapov Sh.A., Fayzullaev J.S.// Offitsialniy vestnik, 2014. - №4.

2. Patent RUz (UZ) №IAP 05432. Magnitouprugiy datchik usiliy/ Amirov S.F., Turdibekov K.Kh., Juraeva K.K., Boltaev O.T., Fayzullaev J.S.// Offitsialniy vestnik, 2017. - №7.

3. Zaripov M.F. Preobrazovateli s raspredelyonnimi parametrami dlya avtomatiki i informatsionnoizmeritelnoy texniki. - Moskva: Energiya, 1969. - 176 s. 4. Konyukhov N.E., Mednikov F.M., Nechaevsky M.L. Elektromagnitnie datchiki mexanicheskix velichin. Moskva: Mashinostoenie, 1987. - 256 s.
5. Bessonov L.A. Teoreticheskie osnovi elektrotexniki. Elektricheskie tsepi. - Moskva: Gardariki, 2006.- 701 s.

6. Sharapov V. Piezoceramic sensors. - Springer, 2011 .-- $498 \mathrm{p}$.

7. Shandrov B.V. Texnicheskie sredstva avtomatizatsii: uchebnik dlya studentov visshix uchebnix zavedeniy/ B.V. Shandrov, A.D. Chudakov. - Moskva: Izdatelskiy tsentr "Academiya", 2007. - $368 \mathrm{~s}$.

8. Zaripov M.F., Zaynullin N.R., Petrova I.Yu. Energoinformatsionniy metod nauchno-texnicheskogo tvorchechstvo. - Moskva: VNIIIPI GKNT, 1988.- 124 s. 9. Golovanova A.M., Kravtsov A.V. Teoreticheskie osnovi elektrotexniki. Elektricheskie izmereniya: Uchebnoe posobie dlya studentov elektrotexnicheskix spetsiolnastey. - Moskva: FGOU VPO MGAU, 2006 .$96 \mathrm{s.}$ 